\title{
Besondere Sitzung vom 29. November 1913.
}

Vorsitzender: Hr. W. Will, Präsident.

Der Vorsitzende heißt den Redner des Abends, Hra. Prof. Dr. The Svedberg, willkommen, ebenso die Vertreter der Physikalischtechnischen Reichsanstalt und des Patentamts und begrüßt unter den zahlreich erschienenen auswärtigen Mitgliedern und Gästen die folgenden HHrn.: O. Allemann (Bern), Dr. Almström (Upsala), Prof. Dr. H. Bechhold (Frankfurt a.M.), Dr. A. Bibergeil (Dessau), Dr. A. Erlenbach (Dessau). Dr. F. W. Frerichs (St. Louis), Dr. B. Hoffmann (Griesheim), Prof. Dr. V. Kohlschütter (Beru), Prof. Dr. A. Lottermoser (Dresden), Dr. W. Mecklenburg (Clausthal), Dr. Wo. Ost. wald (Leipzig), Dr. G. Stalmann (Düsseldorf), Dr. E. Stern (Hannover), Prof. Dr. R. Zsigmondy (Göttingen).

Hr. Prof. Dr. Svedberg erhält nunmebr dạs Wort za seinem zusammenfassenden Vortrage über:

"Die Ergebnisse der Kolloidforschung", an welchen der Vorsitzende die folgende Ansprache anschließt:

^Verehrter Herr Kollege!

Ich bitte Sie, dem Beifall der Gesellschaft auch meinerseits einige Worte des Dankes binzufügen zu dürfen.

Ihre Ausführungen haben uns viel des Interessanten gebracht; sie baben uns ror allem einen an überraschenden Ausblicken reichen Weg zum Stndium der Molekularbewegungen und der Eigenschaften der kleinsten Bausteine unserer natürlichen Welt erschlossen.

Ein Jahrhundert etwa liegen die grundlegenden Arbeiten zurück, die wir Ibrem großen Landsmann Jacob Berzelius verdanken, der zuerst die atomistische Hypothese $z u$ allgemeiner Anerkennung gebracht hat.

Er bat die kritische Sichtung, die experimentelle Vervollkommnung und Prüfung alles dessen, was damals geeignet schien, in dieser Frage

Berichte d. D. Chem. Gesellschaft. Jahrg. XXXXVI. 250 
Aufschluß zu geben, durchgeführt. Von ihm wurde zuerst zusanmenfassend berichtet über all das, was aus den Erfahrungen auf dem Gebiete der Stöchiometrie, der Verbindungsverbältnisse der Gasvolumina, der Elektrochemie, des Isomorphismus, der spezifischen Wärme und andrer $Z$ weige chemiscber Forschung zur Stütze dieser Hypothese berangezogen werden konnte. War sie ibm der vollkommenste Ausdruck der experimentellen Erkenntuis, so bat er doch nicht unterlassen, auf die Unstimmigkeiten aufmerksam zu machen, und wir wollen bei dieser Gelegenheit auch nicht vergessen, welchen Anteil an der Klärung der scheinbaren Widersprüche wir der Aufstellung der lonen-Theorie durch einen weiteren berühmten Kollegen von Hrn. Svedberg, Hrn. Svante Arrhenius, verdanken.

Heute nun hat uns Hr. Svedberg, gestützt auf seine glänzendeu Untersuchungen über die Kolloide und die schönen Arbeiten $Z$ sig mo nd y s und andrer hervorragender Forscher auf diesem Gebiete, gezeigt, wie man molekulare Teilchen und ihre Bewegungen nicht nur mit dem geistigen, sondern auch dem leiblichen Auge verfolgen und messen kann. Damit hat man die auf anderen Wegen ermittelten Gesetze der Molekularwelt von neuem prüfen und bestätigen können, so daß durch diese Untersuchungen die atomistische Anschauung jetzt als ron ibrem hypothetischen Charakter befreit gelten darf, eine Tatsache, die wir als eine stolze Krönung einer hundertjährigen, die Grundlage unserer Wissenschalt betreffenden Arbeit anseben können.

Der Dank, verehrter Hr. Kollege, den Ihnen die Anwesenden gespendet, gilt nicht nur dem schönen Vortrag, der uns das interessante Gebiet so übersichtlich vorgefübrt hat, sondern auch dem hervorragenden Anteil Ihrer Arbeiten an der Förderung unseres Wissens, zu dem ich Sie im Namen unserer Gesellschaft biermit noch herzlichst beglück wünsche."

Der Vorsitzende :

w. Will.
Der Schriftführer:
A. Bannow. 